\title{
Euclidean and Lorentzian Quantum Gravity-Lessons from Two Dimensions
}

\author{
J. AMBJøRN ${ }^{\dagger, *}$, R. LOLL ${ }^{\ddagger .1}$, J. L. NIELSEN ${ }^{\dagger, 2}$ and J. ROLF ${ }^{\dagger, 3}$ \\ † The Niels Bohr Institute, Blegdamsvej 17, DK-2100 Copenhagen $\varnothing$, Denmark \\ ₹ Max-Planck-Institut für Gravitationsphysik, Albert-Einstein-Institut, Schlaatzweg 1, \\ D-14473 Potsdam, Germany
}

\begin{abstract}
No theory of four-dimensional quantum gravity exists as yet. In this situation the twodimensional theory, which can be analyzed by conventional field-theoretical methods, can serve as a toy model for studying some aspects of quantum gravity. It represents one of the rare settings in a quantum-gravitational context where one can calculate quantities truly independent of any background geometry.

We review recent progress in our understanding of $2 \mathrm{~d}$ quantum gravity, and in particular the relation between the Euclidean and Lorentzian sectors of the quantum theory. We show that conventional 2d Euclidean quantum gravity can be obtained from Lorentzian quantum gravity by an analytic continuation only if we allow for spatial topology changes in the latter. Once this is done, one obtains a theory of quantum gravity where space-time is fractal: the intrinsic Hausdorff dimension of usual $2 \mathrm{~d}$ Euclidean quantum gravity is four, and not two. However, certain aspects of quantum space-time remain two-dimensional, exemplified by the fact that its so-called spectral dimension is equal to two. (C) 1999 Elsevier Science Ltd. All rights reserved
\end{abstract}

\section{INTRODUCTION}

Quantum gravity plays havoc with our classical notions of probing the structure of space-time. In classical gravity we determine the metric structure of space-time either by using rods and clocks or by studying the propagation of test particles, from which one can deduce a number of properties of the underlying geometry. When turning to quantum gravity, both methods encounter conceptual and technical difficulties since the object of interest is no longer a single classical space-time, but a quantum average over all possible geometries.

At present there is no quantum gravity theory of four-dimensional Lorentzian metrics which could tell us how to probe the genuine quantum structure of space-time. String theory or recent suggestions of an even more general "M-theory" may eventually tell us how to determine this quantum structure from first principles. However, despite many grand claims they have until now taught us little beyond the standard picture of quantum fluctuations on classical background geometries. Attempts to go further and define genuinely new non-perturbative concepts replacing the classical notions of geometry are at best tentative.

It might thus be useful to try to address questions about the quantum nature of space-time in two dimensions where a conventional quantum field theory of gravity does exist. Such a theory contains no field-theoretical degrees of freedom (it has no gravitons), but only a finite number

\footnotetext{
*e-mail: ambjorn@nbi.dk

${ }^{1}$ e-mail: loll@aei-potsdam.mpg.de

2e-mail: jlnielsen@nbi.dk

3e-mail: rolf@nbi.dk. Now at: Humboldt-Universität, Berlin, Germany.
} 
of quantum-mechanical degrees of freedom. Nevertheless many of the conceptual problems occurring in four dimensions are already present in the two-dimensional theory: how do we define observables invariant under reparametrization? How do we define the propagation of particles? How do we determine simple properties of space-time, in absence of a fixed reference geometry? In a way the quantum nature of these questions is at least as pronounced in $2 \mathrm{~d}$ as it is in $4 \mathrm{~d}$, despite the fact that the action is trivial and non-dynamical. Namely, the triviality of the action implies that all $2 \mathrm{~d}$ geometries have to be included with the same weight in the quantum average. The concept of a fixed background geometry around which one can study small perturbations therefore simply does not exist.

Another moot point in studies of quantum gravity is the question of the signature of the space-time metric. One might hope for the existence of a theory in which the signature was determined dynamically. Even if we are less ambitious and consider the signature as given $a$ priori, nothing much is known about the relation between the quantum theories with Lorentzian and Euclidean signatures. Already classically the simple expedient of applying a Wick rotation $t \rightarrow \tau=i t$ fails in all but a few special cases, for instance, when the space-time is static and thus admits a global choice of time $x_{0}$ such that the cross terms $g_{0 i}, i \geq 1$ of the metric tensor vanish and its spatial components $g_{i j}$ are time-independent. We do not know of a way to set up a 1-to1 correspondence between generic solutions of the continuum Einstein equations with different signatures. If it exists, it is likely to be technically involved. From this point of view it is unclear if one can expect to obtain the correct Lorentzian theory by first performing a path integral over general Euclidean metric configurations and then analytically continuing in some way, as is the case in conventional field theory in flat space-time. Again, two-dimensional quantum gravity is a good laboratory for the study of such questions, since the theory can be treated by conventional field-theoretical tools, and turns out to be non-perturbatively renormalizable.

In the next section we briefly describe a quantum gravity analogue of the classical rods-andclocks measurement, that is, how to extract diffeomorphism-invariant information about the geometry of quantum space-time. Section 3 contains some details of a discussion of test particle propagation in quantum space-time. We then define a path-integral quantization of metric spacetimes with Lorentzian signature and show that the fractal structure of space-time only agrees with the one obtained in Euclidean gravity if topology changes of space are included. The final section contains a discussion of the results and some remarks on possible generalizations to higher dimensions.

\section{THE FUNCTIONAL INTEGRAL OVER 2D GEOMETRIES}

The partition function for two-dimensional Euclidean gravity is given by

$$
Z(\Lambda)=\int \mathcal{D}[g] \mathrm{e}^{-\Lambda V_{g}}, \quad V_{g} \equiv \int_{\mathcal{M}} \mathrm{d}^{2} \xi \sqrt{\operatorname{det} g(\xi)},
$$

where $\mathcal{D}[g]$ denotes a suitable measure on the space of equivalence classes $[g]$ of Riemannian two-metrics, and $\Lambda$ is the bare cosmological constant. The weight factor in the exponential is proportional to the Einstein action, which in the present case consists only of the cosmological term. (The scalar curvature term has been omitted since it gives only a constant contribution for fixed space-time topology.) It is sometimes convenient to consider the partition function where the volume $V$ of space-time is kept fixed. We define it by

$$
Z(V)=\int \mathcal{D}[g] \delta\left(V-V_{g}\right)
$$

such that 


$$
Z(\Lambda)=\int_{0}^{\infty} \mathrm{d} V \mathrm{e}^{-V \Lambda} Z(V)
$$

In the following we will discuss a set of reparametrization-invariant "observables", namely, the Hartle-Hawking wave functionals and the two-point functions of the theory. The HartleHawking wave functional is defined by

$$
W_{\Lambda}(L)=\int \mathcal{D}[g] \mathrm{e}^{-S(g ; \Lambda)},
$$

where $L$ stands for the metric degrees of freedom on the boundary $\partial \mathcal{M}$ of the manifold $\mathcal{M}$. In $2 d$ the equivalence class of the boundary metric (in the case of one boundary component) is uniquely fixed by its length, which we will again denote by $L$. In dimensions higher than two, instead of the single parameter $L$ one would need to specify a metric equivalence class $\left[\left.g\right|_{\partial M}\right]$ on $\partial \mathcal{M}$. The functional integration would then have to be performed over all metric configurations in the interior which induce $\left[\left.g\right|_{\partial M}\right]$ on the boundary.

It is often convenient to consider boundaries with variable length $L$ by introducing a boundary cosmological term in the action, resulting in

$$
S(g ; \Lambda, X)=\Lambda \int_{\mathcal{M}} \mathrm{d}^{2} \xi \sqrt{\operatorname{det} g(\xi)}+X \int_{\partial \mathcal{M}} \mathrm{d} s,
$$

where $\mathrm{d} s$ is the invariant line element induced by $g$ on the boundary and $X$ is called the boundary cosmological constant. We may then define

$$
W_{\Lambda}(X)=\int \mathcal{D}[g] \mathrm{e}^{-S(g ; \Lambda, X)}
$$

so that the wave-functions $W_{\Lambda}(L)$ and $W_{\Lambda}(X)$ are related by a Laplace transformation in the boundary length,

$$
W_{\Lambda}(X)=\int_{0}^{\infty} \mathrm{d} L \mathrm{e}^{-X L} W_{\Lambda}(L) .
$$

The two-point function $G_{\Lambda}(R)$ is defined by

$$
G_{\Lambda}(R)=\int \mathcal{D}[g] \mathrm{e}^{-S(g, \Lambda)} \iint \mathrm{d}^{2} \xi \sqrt{\operatorname{det} g(\xi)} \mathrm{d}^{2} \eta \sqrt{\operatorname{det} g(\eta)} \delta\left(D_{g}(\xi, \eta)-R\right),
$$

where $D_{g}(\xi, \eta)$ denotes the geodesic distance between the points $\xi$ and $\eta$ in the given metric $g_{a b}$. Again, it is sometimes convenient to consider a situation where the space-time volume $V$ is fixed. The corresponding function $G_{V}(R)$ will be related to (8) by a Laplace transformation, analogous to the partition functions $Z$ above,

$$
G_{\Lambda}(R)=\int_{0}^{\infty} \mathrm{d} V \mathrm{e}^{-V \Lambda} G_{V}(R)
$$

Both $G_{\Lambda}(R)$ and $G_{V}(R)$ have the interpretation of partition functions for universes with pairs of marked points separated by a given geodesic distance $R$. If we denote the average volume of a spherical shell of geodesic radius $R$ in the class of metrics with space-time volume $V$ by $S_{V}(R)$, we have by definition

$$
S_{V}(R)=\frac{G_{V}(R)}{V Z(V)}
$$


The function $S_{V}(R)$ therefore serves as the quantum analogue of the volume of a classical spherical shell of radius $R$ in a fixed geometry. In this sense it is the quantum version of a "rods-and-clocks" measurement of the geometry. In the same spirit one may define an intrinsic fractal dimension $d_{H}$ of the ensemble of metrics by the scaling behaviour

$$
\lim _{R \rightarrow 0} S_{V}(R) \sim R^{d_{H}-1}(1+O(R)) .
$$

Alternatively, one could introduce another notion of dimensionality $d_{H}$ via

$$
\langle V\rangle_{R} \sim-\frac{\partial \log G_{\Lambda}(R)}{\partial \Lambda} \sim R^{d_{H}},
$$

for a suitable range of $R$ related to the value of $\Lambda$. As will become clear, these two definitions agree in the case of pure gravity. Eq. (11) can be viewed as a "local" definition of $d_{H}$, while eqn (12) characterizes a "global" property of space-time. Since the two definitions result in the same value for $d_{H}$, two-dimensional gravity can be said to have a genuine fractal dimension over all scales,

In the regularization we will be using each discrete geometry, representing an equivalence class of metrics $[g]$, will be included with the same weight. The functional integral $\int \mathcal{D}[g]$ is turned into a discrete sum over inequivalent geometries, each with weight one. Eq. (2) then entails that the calculation of $Z(V)$ basically amounts to a counting problem. The same is true for the other observables defined above. One way of performing the summations is to introduce a suitable regularization of the set of geometries by means of a cut-off, perform the summation, and then to remove the cut-off.

\subsection{The regularization}

In the calculations referred to below we regularize the integral over geometries by using equilateral triangles as elementary building blocks, which are glued together in all possible ways consistent with a given space-time topology [1-4]. The metric degrees of freedom are encoded in the different ways of gluing the triangles. For example, the deficit angle at a vertex $v$ of the triangulation (a measure of local curvature) is determined by the number of triangles meeting at $v$. From this point of view a summation over such triangulations forms a grid in the class of Riemannian geometries associated with a given manifold $\mathcal{M}$. The hope is that in the limit as the edge-length $a$ of the triangles is taken to zero, this grid becomes sufficiently dense and uniform to approximate correctly the functional integral over all Riemannian geometries. We will provide evidence that this is the case by explicit calculations that are in agreement with the corresponding continuum expressions, where they can be compared.

The surprising situation encountered in two-dimensional quantum gravity is the fact that the analytical power of the regularized theory seems to exceed that of the formal continuum manipulations performed within Liouville theory. This reverses the usual situation where regularized theories are either used in a perturbative context to remove infinities order by order, or introduced in a non-perturbative setting to make numerical simulations possible. By contrast, in the regularized framework of $2 \mathrm{~d}$ quantum gravity at hand, we will derive analytic (continuum) expressions with an ease which can presently not be matched by formal continuum manipulations.

\subsection{The two-loop and two-point functions}

We first define a generalization of the two-point function: consider universes with the topology of a finite cylinder, where the exit loop (the outgoing $S^{l}$-boundary component) is separated from the entrance loop (the incoming $S^{1}$-boundary) by a geodesic distance $R$. By definition this 
means that each point on the exit loop has a geodesic distance $R$ to the entrance loop (but not necessarily the converse). The partition function for such universes will be denoted by

$$
G_{\Lambda}\left(L_{1}, L_{2} ; R\right),
$$

where $L_{1}$ and $L_{2}$ are the lengths of the entrance and exit loops. Introducing boundary cosmological constants $X$ and $Y$ for the entrance and exit loops we obtain

$$
G_{\Lambda}(X, Y ; R)=\int_{0}^{\infty} d L_{1} \int_{0}^{\infty} d L_{2} \mathrm{e}^{-L_{1} X} \mathrm{e}^{-L_{2} Y} G_{\Lambda}\left(L_{1}, L_{2} ; R\right)
$$

by a Laplace transformation.

In section 4 we will describe our regularized model in some detail, but for the moment we are only interested in some general aspects independent of those details. In a regularized model we must introduce dimensionless coupling constants $\lambda_{b_{1}}, \lambda_{b_{2}}$ and $\lambda$ corresponding to the bare couplings $X, Y$ and $\Lambda$. Since $X(Y)$ and $\Lambda$ have dimensions of mass and (mass) ${ }^{2}$, we expect additive renormalizations of the form

$$
\lambda=\lambda_{c}+a^{2} \Lambda, \quad \lambda_{b_{1}}=\lambda_{b}^{c}+a X, \quad \lambda_{b_{2}}=\lambda_{b}^{c}+a Y .
$$

For convenience we introduce the notation

$$
g=\mathrm{e}^{-\lambda}=g_{c}\left(1-\frac{1}{2} a^{2} \Lambda\right), \quad x=\mathrm{e}^{-\lambda_{b_{1}}}=x_{c}(1-a X), \quad y=\mathrm{e}^{-\lambda_{b_{2}}}=x_{c}(1-a Y) .
$$

Strictly speaking this involves an analytic coupling constant redefinition of $X, Y$, compared to (15). In this way we obtain regularized two-loop functions $G_{\lambda}(x, y ; r)$ and $G_{\lambda}\left(l_{1}, l_{2} ; r\right)$, where the integer $r$ is the geodesic distance measured in lattice units, and $l_{1}, l_{2}$ are the lengths of the entrance and exit loops, again measured in lattice units. $G_{\lambda}(x, y ; r)$ will be related to $G_{\lambda}\left(l_{1}, l_{2} ; r\right)$ by the discretized analogue of (14), namely,

$$
G_{\lambda}(x, y ; r) \equiv \sum_{k, l} x^{k} y^{\prime} G_{\lambda}(k, l ; r)
$$

illustrating that from a combinatorial point of view $G_{\lambda}(x, y ; r)$ can be regarded as the generating functional for the numbers $G_{\lambda}\left(l_{1}, l_{2} ; r\right)$.

The two-loop function satisfies the combinatorial identity

$$
G_{\lambda}\left(l_{1}, l_{2}, r_{1}+r_{2}\right)=\sum_{l} G_{\lambda}\left(l_{1}, l ; r_{1}\right) G_{\lambda}\left(l, l_{2} ; r_{2}\right)
$$

and in particular

$$
G_{\lambda}\left(l_{1}, l_{2} ; r+1\right)=\sum_{l} G_{\lambda}\left(l_{1}, l ; 1\right) G_{\lambda}\left(l, l_{2} ; r\right)
$$

The function $G_{\lambda}\left(l_{1}, l_{2} ; 1\right)$ appears on the right-hand side as a transfer matrix, and knowing $G_{\lambda}\left(l_{1}, l_{2} ; 1\right)$ allows us to find $G_{\lambda}\left(l_{1}, l_{2} ; r\right)$ by iterating $(19) r$ times. As usual in combinatorics, this program is conveniently carried out by means of the generating function $G_{\lambda}(x, y ; r)$ for the numbers $G_{\lambda}\left(l_{1}, l_{2} ; r\right)$. We rewrite relation $(18)$ as

$$
G_{\lambda}\left(x, y ; r_{1}+r_{2}\right)=\oint \frac{d z}{2 \pi i z} G_{\lambda}\left(x, z^{-1} ; r_{1}\right) G_{\lambda}\left(z, y ; r_{2}\right)
$$

where the contour should be chosen to include the singularities in the complex $z$-plane of $G_{\lambda}\left(x, z^{-1} ; r_{1}\right)$ but not those of $G_{\lambda}\left(z, y ; r_{2}\right)$. 


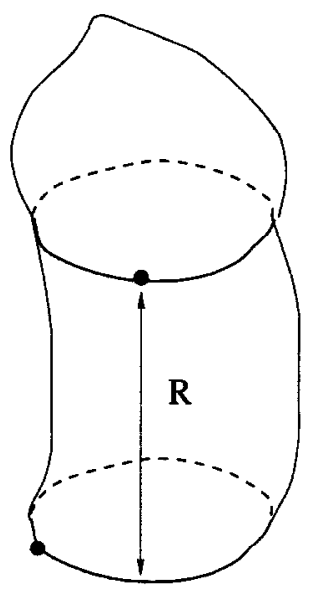

Fig. 1. Marking a vertex in the bulk of $W_{\Lambda}(X)$. The vertex has a distance $R$ from the boundary loop, which has one marked vertex

In the same way we can define the regularized Hartle-Hawking wave functionals $w_{\lambda}(l)$ and $w_{\lambda}(x)$ corresponding to $W_{\Lambda}(L)$ and $W_{\Lambda}(X)$. The former, $w_{\lambda}(l)$, will be a sum over an appropriate class of triangulations with the topology of a disc and a boundary of lattice length $l$, and the continuum relation (7) replaced by

$$
w_{\lambda}(x)=\sum_{l} x^{l} w_{\lambda}(l)
$$

While the scaling of $G_{\lambda}(x, y ; r)$,

$$
G_{\lambda}(x, y ; r) \rightarrow a^{-1} G_{\Lambda}(X, Y ; R) \text { for } a \rightarrow 0,
$$

follows directly from $(20)$ and (15), we will assume that $w_{\lambda}(x)$ has the general form

$$
w_{\lambda}(x)=w_{\lambda}^{n s}(x)+a^{\eta} W_{\Lambda}(X)+\text { less singular terms. }
$$

We now introduce an explicit mark in the bulk of $w_{\lambda}(x)$ by differentiating with respect to $\lambda$. This leads to the combinatorial identity

$$
\frac{\partial w_{\lambda}(x)}{\partial \lambda}=\sum_{r} \sum_{l} G_{\lambda}(x, l ; g ; r) l w_{\lambda}(l),
$$

which after a Laplace transformation becomes

$$
\frac{\partial w_{\lambda}(x)}{\partial \lambda}=\sum_{r} \oint \frac{d z}{2 \pi i z} G_{\lambda}\left(x, z^{-1} ; r\right) \frac{\partial w_{\lambda}(z)}{\partial z} .
$$

This is illustrated in Fig. 1. A given mark has a distance $r$ ( $R$ in the continuum) to the entrance loop. In the figure we have drawn all points with the same distance to the entrance loop and which form a connected loop containing the marked point. Let us now assume a general scaling of the form

$$
R=a^{\varepsilon} r, \quad \varepsilon>0,
$$

for the geodesic distance $R$ in the continuum limit. Inserting eqns (25) and (26) into (24) we obtain

$$
\frac{\partial w_{\lambda}^{n s}}{\partial \lambda}-a^{\eta-2} \frac{\partial W_{\Lambda}(X)}{\partial \Lambda}=\frac{1}{a^{\varepsilon}} \int d R \int d Z G_{\Lambda}(X,-Z ; R)\left[\frac{\partial w_{\lambda}^{n s}}{\partial z}-a^{n-1} \frac{\partial W_{\Lambda}(Z)}{\partial Z}\right],
$$


where $(x, \lambda)=\left(x_{c}, \lambda_{c}\right)$ in the non-singular part.

It follows from eqn (27) that the only consistent choices for $\eta$ are either $\eta<0$ (in which case $\varepsilon=1$ ) or $1<\eta<2$. We will presently consider the latter situation, and later in section 4 obtain an interpretation for the case $\eta<0, \varepsilon=1$. For $1<\eta<2$, eqn (27) splits into the two relations

$$
-a^{\eta-2} \frac{\partial W_{\Lambda}(X)}{\partial \Lambda}=\left.\frac{1}{a^{\varepsilon}} \frac{\partial w_{n s}}{\partial z}\right|_{z=x_{c}} \int d R \int d Z G_{\Lambda}(X,-Z ; R)
$$

and

$$
\left.\frac{\partial w_{n s}}{\partial g}\right|_{g=g_{c}}=-\frac{a^{\eta-1}}{a^{\varepsilon}} \int d R \int d Z G_{\Lambda}(X,-Z ; R) \frac{\partial W_{\Lambda}(Z)}{\partial Z} .
$$

We are led to the conclusion that $\varepsilon=1 / 2$ and $\eta=3 / 2$. This implies that the geodesic distance $R$ has an anomalous dimension. Note for future reference that eqn (28) is of the form

$$
-\frac{\partial W_{\Lambda}(X)}{\partial \Lambda}=\text { const. } G_{\Lambda}\left(X, L_{2}=0\right),
$$

where $G_{\Lambda}\left(L_{1}, L_{2}\right)$ is a (generalized) Hartle-Hawking wave functional with two boundaries, the cylinder amplitude

$$
G_{\Lambda}\left(L_{1}, L_{2}\right)=\int_{0}^{\infty} d R G_{\Lambda}\left(L_{1}, L_{2} ; R\right)
$$

The disc amplitude $W_{\Lambda}(X)$ can be calculated explicitly using either combinatorial techniques (see [19] for a review) or matrix model methods ([13,14]), and is found to have a dimension corresponding to $\eta=3 / 2$. Above we have reproduced this non-trivial result from simple combinatorial arguments. These can be taken further within the dynamical triangulations approach to calculate explicitly the transfer matrix $G_{\lambda}(x, y ; 1)$ (see [6] or [19] for details), which appears in eqn (20) in the special case when $r_{1}=1$,

$$
G_{\lambda}(x, y ; r+1)=\oint \frac{d z}{2 \pi i z} G_{\lambda}\left(x, z^{-1} ; 1\right) G_{\lambda}(z, y ; r)
$$

An even simpler (and at the same time more general) derivation of the corresponding continuum equation will be given in section 4 below, in the context of a slightly different model. Since twodimensional Euclidean quantum gravity corresponds to $\eta=3 / 2, \varepsilon=1 / 2$, we can substitute these values into eqn (83) below to derive the equation

$$
\frac{\partial}{\partial R} G_{\Lambda}\left(X, L_{2} ; R\right)=-\frac{\partial}{\partial X}\left[W_{\Lambda}(X) G_{\Lambda}\left(X, L_{2} ; R\right)\right]
$$

for $G_{\Lambda}\left(X, L_{2}, R\right)$ (which also could have been obtained directly from (32)). Eq. (33) determines the structure of two-dimensional Euclidean quantum gravity. First, it allows us to calculate $W_{\Lambda}(X)$, the Hartle-Hawking wave functional. Integrating (33) with respect to $R$ and using relation (30) as well as the boundary condition $G_{\Lambda}\left(X, L_{2}=0 ; R=0\right)=1$ (originating from $\left.G_{\Lambda}\left(L_{1}, L_{2} ; R=0\right)=\delta\left(L_{1}-L_{2}\right)\right)$, we obtain

$$
-1=\frac{\partial}{\partial X}\left[W_{\Lambda}(X) \frac{\partial}{\partial \Lambda} W_{\Lambda}(X)\right]
$$

Since $W_{\Lambda}(X)$ has length dimension $-3 / 2(\eta=3 / 2)$, i.e. $W_{\Lambda}^{2}(X)=X^{3} F(\sqrt{\Lambda} / X)$, the general solution must be of the form

$$
W_{\Lambda}(X)=\sqrt{-2 \Lambda X+b^{2} X^{3}+c^{2} \Lambda^{3 / 2}}
$$


From the very origin of $W_{\Lambda}(X)$ as the Laplace transform of the Hartle-Hawking wave functional $W_{\Lambda}(L)$ which is bounded, it follows that $W_{\Lambda}(X)$ has no singularities or cuts for $\operatorname{Re} X>0$. This leads, after a suitable rescaling of the cosmological constant $X$, to the expression

$$
W_{\Lambda}(X)=\left(X-\frac{1}{2} \sqrt{\Lambda}\right) \sqrt{X+\sqrt{\Lambda}}
$$

well-known from much more complicated calculations using matrix models and dynamical triangulations. Once we know $W_{\Lambda}(X)$, we can solve eqn (33) and obtain $G_{\Lambda}(R)$ as the integral

$$
G_{\Lambda}(R)=\int \mathrm{d} L_{2} G\left(L_{1}=0, L_{2} ; R\right) L_{2} W_{\Lambda}\left(L_{2}\right)
$$

This combinatorial identity is again illustrated in Fig. 1. Contracting $L_{1}$ to zero corresponds to introducing a marked point. Let the other marked point be at geodesic distance $R$. It will be located at a loop of some length $L_{2}$, having a distance $R$ from the first marked point. All such manifolds are obtained by closing the loop with length $L_{2}$ by a "cap" of length $L_{2}$ and integrating over all $L_{2}$. This is precisely the combinatorial content of expression (37). By a direct calculation one obtains

$$
G_{\Lambda}(R)=\Lambda^{3 / 4} \frac{\cosh [R \sqrt[4]{\Lambda}]}{\sinh ^{3}[R \sqrt[4]{\Lambda}]}
$$

Using this expression it follows that

$$
\langle V\rangle_{R} \sim-\frac{\partial \log G_{\Lambda}(R)}{\partial \Lambda} \sim R^{4}\left(1+O\left(R^{4} \Lambda\right)\right),
$$

implying that $d_{H}=4$ rather than 2 , as one naively would have expected. Observe also that $G_{\Lambda}(R)$ falls off exponentially for $R \gg \sqrt[4]{\Lambda}$. It has been shown that this is a consequence of the same kind of sub-additivity arguments which lead to the exponential fall-off of the massive particle propagator [15].

The quantity $d_{H}$ above is the "globally defined" Hausdorff dimension in the sense discussed below (12), as is clear from eqn (38). We can determine the "local" dimension $d_{H}$, defined by eqn (11), by performing the inverse Laplace transformation of $G_{\Lambda}(R)$ to obtain $G_{V}(R)$. The average volume $S_{V}(R)$ of a spherical shell of geodesic radius $R$ in the ensemble of universes with space-time volume $V$ can then be calculated from relation (10), yielding

$$
S_{V}(R)=R^{3} F\left(R / V^{\frac{1}{4}}\right), \quad F(0)>0,
$$

where $F(x)$ can be expressed in terms of certain generalized hypergeometric functions [16]. Eq. (40) shows that also the "local" dimension $d_{H}$ is equal to four.

\subsection{Summary}

It has been shown how one can calculate the functional integral over two-dimensional geometries, by proceeding in close analogy with the functional integral over random paths. One of the fundamental results of the latter theory is that a generic random path between two points in $R^{d}$, separated a geodesic distance $R$, is not proportional to $R$ but to $R^{2}$. This famous result has a direct translation to the theory of random two-dimensional geometries: the generic volume of a closed universe of radius $R$ is not proportional to $R^{2}$ but to $R^{4}$. 


\section{THE SPECTRAL DIMENSION OF SPACE-TIME}

In the preceding section we have described a quantum version of the classical measurements of space-time geometry. We now turn to the test particle aspect. Consider the propagation of test particles in a fixed geometry. By observation we can determine their propagator, and by an inverse Laplace transformation the associated heat kernel (see below). The heat kernel has an asymptotic expansion whose individual terms are functions of various contractions of the Riemann tensor and other geometric quantities, and thus have a direct geometric interpretation. One may wonder how much of this information survives in a genuine theory of quantum gravity where we integrate over all geometries. Such a study leads naturally to the notion of spectral dimension as a measure of the structure of quantum space-time.

The most intuitive definition of the spectral dimension is based on the diffusion equation on a (compact) manifold with metric $g_{a b}$. Let $\Delta_{g}$ denote the Laplace-Beltrami operator corresponding to $g_{a h}$. The probability distribution $K\left(\xi, \xi^{\prime} ; \tau\right)$ of diffusion in a fictitious "time" $\tau$ is related to the massless scalar propagator $\left(-\Delta_{g}\right)^{-1}$ by ${ }^{\dagger}$

$$
\left\langle\xi^{\prime}\left|\left(-\Delta_{g}\right)^{-1}\right| \xi\right\rangle^{\prime}=\int_{0}^{\infty} \mathrm{d} \tau K^{\prime}\left(\xi, \xi^{\prime} ; \tau\right) .
$$

In particular, the average return probability distribution at "time" $T$ has the small- $T$ behaviour

$$
R P_{g}^{\prime}(\tau) \equiv \frac{1}{V_{g}} \int \mathrm{d}^{d} \xi \sqrt{\operatorname{det} g} K^{\prime}(\xi, \xi ; \tau) \sim \frac{1}{\tau^{d / 2}}(1+O(\tau))
$$

where $V_{g}$ denotes the volume of the compact manifold with metric $g$. The important point in the context of quantum gravity is that $R P_{g}^{\prime}(\tau)$ is invariant under reparametrization. We may therefore define the quantum average over geometries,

$$
R P_{V}^{\prime}(\tau) \equiv \frac{1}{Z_{V}} \int \mathcal{D}[g]_{V} \mathrm{e}^{-S_{\mathrm{ef}}([g])} R P_{g}^{\prime}(\tau)
$$

where $S_{\text {eff }}([g])$ denotes the effective action of quantum gravity after the integration of possible matter fields, and $Z_{V}$ denotes the partition function of quantum gravity plus matter for a fixed space-time volume $V$. The spectral dimension $d_{s}$ in quantum gravity is now defined by the small$\tau$ behaviour of the functional average $R P_{V}^{\prime}(\tau)$,

$$
R P_{V}^{\prime}(\tau) \sim \frac{1}{\tau^{d_{s} / 2}}(1+O(\tau))
$$

The $O(\tau)$ term in (42) has a well-known asymptotic expansion in powers of $\tau$, where the coefficient of $\tau^{r}$ is an integral over certain powers and contractions of the curvature tensor. This asymptotic expansion breaks down when $T \sim V^{2 / d}$ at which point the exponential decay in $T$ of the heat kernel $K$ takes over. If we average over all geometries as in (43), it is natural to expect that the only invariant left will be the volume $V$ which is kept fixed. Thus we expect a relation of the form

$$
R P_{V}^{\prime}(\tau)=\frac{1}{\tau^{d_{s} / 2}} F\left(\frac{T}{V^{2 / d_{s}}}\right)
$$

where $F(0)>0$ and $F(x)$ falls off exponentially fast for $x \rightarrow \infty$.

\footnotetext{
† Since we consider compact manifolds, the Laplace-Beltrami operator $\Delta_{g}$ has zero modes. Eq. (41) should be understood with these zero modes projected out. This is indicated by the prime.
} 
For a fixed manifold of dimension $d$ and a given smooth geometry [ $g$ ] we have $d=d_{s}$ by definition. The functional average can in principle change this value, i.e. the dimension of $\tau$ may become anomalous. A well-known example of a similar nature can be found for the ordinary free particle. In the path-integral representation of the free particle, any smooth path has of course fractal dimension one. Nevertheless the short-distance properties of the free particle reflect the fact that the generic path contributing to the path integral has fractal dimension (the extrinsic Hausdorff dimension in the target space $R^{D}$ ) $D_{H}=2$ with probability one. In the same way the functional integral over geometries might change $d_{s}$ from the "naive" value $d$. In two-dimensional quantum gravity it is known, as mentioned above, that the intrinsic Hausdorff dimension is different from $d=2$ and the generic geometry is in this sense fractal, with probability one. When one considers diffusion on fixed fractal structures (often embedded in $R^{D}$ ), it is well-known that $d_{s}$ can be different from both $D$ and the fractal dimension $d_{h}$ of the structure. If $\delta$ denotes the so-called anomalous gap exponent, defined by the relation between the diffusion time $\tau$ and the average spread of diffusion on the fractal structure, but measured in $R^{D}$,

$$
\left\langle r^{2}(\tau)\right\rangle \sim \tau^{2 / \delta}
$$

the relation between the fractal dimension (intrinsic Hausdorff dimension) $d_{h}$ of the structure, the spectral dimension of the diffusion and the gap exponent is given by

$$
d_{s}=\frac{2 d_{h}}{\delta}
$$

If $\delta$ is not anomalous, i.e. $\delta=2$ as for diffusion on a smooth geometry, we have $d_{s}=d_{h}$, which is the analogue of $d_{s}=d$ for fixed smooth geometries. However, in general $\delta \neq 2$ (for a review of diffusion on fractal structure, see, for example, [17]).

\subsection{The spectral dimension of $2 d$ quantum gravity}

Let us now present a proof that $d_{s}=2$ in two-dimensional Euclidean gravity coupled to conformal matter with a central change $c \leq 1$ (i.e. for the range of conformal theories where Liouville field theory is applicable to $2 \mathrm{~d}$ quantum gravity coupled to matter).

Since $\Delta_{\lambda g}=\lambda^{1} \Delta_{g}$, we have from definition (41) that

$$
\operatorname{Tr}^{\prime}\left(-\Delta_{\lambda g}\right)^{-1}=\lambda \operatorname{Tr}^{\prime}\left(-\Delta_{g}\right)^{-1}
$$

According to Liouville theory applied to two-dimensional quantum gravity coupled to a conformal field theory with central charge $c$, a general spin-less operator, $\Phi_{n}[g]$, which depends only on the metric $g_{a b}$, which is diffeomorphism-invariant, and which satisfies $\Phi[\lambda g]=\lambda^{-n} \Phi_{n}[g]$ at the classical level, has the following scaling of its quantum expectation value ${ }^{+}$(see [5] for details):

$$
\left\langle\Phi_{n}[g]\right\rangle_{\lambda \nu}=\lambda^{\alpha_{-n} / \alpha_{1}}\left\langle\Phi_{n}[g]\right\rangle_{V}, \quad \alpha_{n}=\frac{2 n \sqrt{25-c}}{\sqrt{25-c}+\sqrt{25-c-24 n}}
$$

\footnotetext{
t The expectation value $\left\langle\Phi_{n}[g]\right\rangle_{V}$ of this operator in the context of two-dimensional quantum gravity coupled to a conformal field theory with central charge $c$ is defined by$$
\left\langle\Phi_{n}[g]\right\rangle_{V}=\frac{1}{Z_{V}} \int \mathcal{D}[g]_{V} \mathrm{e}^{-S_{e f f}[g]} \Phi_{n}[g]
$$

where $e^{-S_{e f f}[g]}$ again denotes the effective action after integration over the matter fields, and $\left.Z_{V} \mid g\right]$ denotes the corresponding partition function for fixed space-time volume $V$.
} 
It follows that the quantity

$$
\frac{1}{V}\left\langle\operatorname{Tr}^{\prime}\left(-\Delta_{g}\right)^{-1}\right\rangle_{V}
$$

is invariant under the transformation $V \rightarrow \lambda V$. On the other hand, the assumed scaling (45) implies that

$$
\begin{aligned}
\frac{1}{V}\left\langle\operatorname{Tr}^{\prime}\left(-\Delta_{g}\right)^{-1}\right\rangle_{V} & =\int_{0}^{\infty} \mathrm{d} \tau R P^{\prime}(\tau)=\int_{0}^{\infty} \mathrm{d} \tau \frac{1}{\tau^{d_{s} / 2}} F\left(\frac{\tau}{V^{2} / d_{s}}\right) \\
& =\text { const. } \times V^{2 / d_{s}-1} .
\end{aligned}
$$

Thus it follows that $d_{s}=2$.

To corroborate this result, let us use the same kind of argument to derive a simple relation between the spectral dimension and the extrinsic Hausdorff dimension for dynamical self-similar systems like random surface models, branched polymers etc. (see [10] for details, for a definition of branched polymer models see $[12,18])$. Since the extrinsic Hausdorff dimension is known for these systems it will allow a determination of the spectral dimension. As a typical example of such models (and maybe the most interesting), we consider random surfaces, here described as two-dimensional quantum gravity coupled to $D$ Gaussian fields $X_{\mu}$. These fields can be viewed as embedding coordinates for a surface in $R^{D}$. The partition function for such a system is given by

$$
Z_{V}=\int \mathcal{D}[g]_{V} \mathcal{D}\left[X_{\mu}\right]_{\mathrm{cm}} \mathrm{e}^{-\int \mathrm{d}^{2} \xi \sqrt{\operatorname{det} g} g^{a b} \partial_{a} X_{\mu} \partial_{b} X_{\mu}}
$$

Here $\mathcal{D}\left[X_{\mu}\right]_{\mathrm{cm}}$ denotes the functional integration over the $D$ Gaussian fields $X_{\mu}$, but with the center of mass fixed (to zero). The extrinsic Hausdorff dimension $D_{H}$ is usually defined as

$$
\left\langle X^{2}\right\rangle_{V} \sim V^{2 / D_{H}} \text { for } V-\infty,
$$

where

$$
\left\langle X^{2}\right\rangle_{V} \equiv \frac{1}{Z_{V}} \int \mathcal{D}[g]_{V} \mathcal{D}\left[X_{\mu}\right]_{\mathrm{cm}} \mathrm{e}^{-\int \mathrm{d}^{2} \xi \sqrt{\operatorname{det} g} g^{\alpha h} \partial_{a} X_{\mu} \partial_{b} X_{\mu}}\left(\frac{\int \mathrm{d}^{2} \xi \sqrt{\operatorname{det} g} X_{\mu}^{2}(\xi)}{D V}\right)
$$

The Gaussian action in $X$ implies that

$$
\begin{aligned}
\left\langle X^{2}\right\rangle_{V} & =\left.\frac{1}{D V Z_{V}} \frac{\partial}{\partial \omega} \int \mathcal{D}[g]_{V} \mathcal{D}\left[X_{\mu}\right]_{\mathrm{cm}} \mathrm{e}^{-\int \mathrm{d}^{2} \xi \sqrt{\operatorname{det} g} g^{\alpha b} \partial_{a} X_{\mu} \partial_{h} X_{\mu}+\omega \int \mathrm{d}^{2} \xi \sqrt{\operatorname{det} g} X_{\mu}^{2}(\xi)}\right|_{\omega=0} \\
& =\left.\frac{1}{D V Z_{V}} \frac{\partial}{\partial \omega} \int \mathcal{D}[g]_{V}\left(\operatorname{det}^{\prime}\left(-\Delta_{g}-\omega\right)\right)^{-D / 2}\right|_{\omega=0} \\
& =\frac{1}{2 V Z_{V}} \int \mathcal{D}[g]_{V}\left(\operatorname{det}^{\prime}\left(-\Delta_{g}\right)\right)^{-D / 2} \operatorname{Tr}^{\prime}\left[\frac{1}{-\Delta_{g}}\right] \\
& =\frac{1}{2 V}\left\langle\operatorname{Tr}^{\prime}\left[\frac{1}{-\Delta_{g}}\right]\right\rangle_{V}
\end{aligned}
$$

where the primes on the determinants and traces again mean that zero modes are excluded. Formula (54) is used to define $\left\langle X^{2}\right\rangle_{V}$ when $D$ is non-integer. From (50) we obtain

$$
\left\langle X^{2}\right\rangle_{V} \sim V^{2 / d_{s}-1}
$$




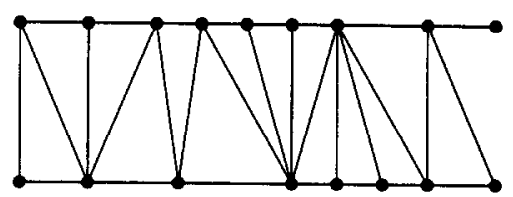

Fig. 2. The propagation of a spatial slice from step $t$ to step $t+1$. The ends of the strip should be joined to form a band with topology $S^{1} \times[0,1]$.

for $V$ going to infinity, and from (52) we now conclude that

$$
\frac{1}{d_{s}}=\frac{1}{D_{H}}+\frac{1}{2}
$$

Since it is well-known that $D_{H}=\infty$ for $D$ Gaussian fields coupled to 2 d quantum gravity [20], as long as $D \leq 1$ we reach again the conclusion that $d_{s}=2$ for such systems.

Note that the scaling relation (56) is valid for other self-similar systems like branched polymers. For the branched polymer systems we have $D_{H}=4$ and we are led to the known, but non-trivial result that $d_{s}=4 / 3$, the famous Alexander-Orbarch value, in the case of branched polymers [8]. Formula (56) also applies for so-called multicritical branched polymers [18], where $D_{H}=$ $2 m /(m-1), m \geq 2$.

\section{LORENTZIAN SIGNATURE AND CAUSAL STRUCTURES}

As mentioned in the introduction, the solution of two-dimensional quantum gravity via an intermediate discretization amounts to the counting of inequivalent geometries. While this counting problem has been solved in Euclidean gravity by the use of dynamical triangulations, an extension to space-times with Lorentzian signature is not entirely straightforward. We propose here a discretized model, formulated in the spirit of dynamical triangulations, where a causal structure - characteristic for Lorentzian space-time metrics - is explicitly present in all the geometries included in the path integral.

The model is defined as follows. The topology of the underlying manifold is taken to be $S^{1} \times[0,1]$, with "space" represented by the closed manifold $S^{1}$. We consider the evolution of this space in "time". For the moment, no spatial topology changes are allowed, but we will return to this issue later.

The geometry of each spatial slice is uniquely characterized by the length assigned to it. In the discretized version, the length $L$ will be quantized in units of a lattice spacing $a$, i.e. $L=l \cdot a$ where $l$ is an integer. A slice will thus be defined by $l$ vertices and $l$ links connecting them. To obtain a $2 d$ geometry, we will evolve this spatial loop in discrete steps. This leads to a preferred notion of (discrete) "time" $t$, where each loop represents a slice of constant $t$. The propagation from time-slice $t$ to time-slice $t+1$ is governed by the following rule: each vertex $i$ at time $t$ is connected to $k_{i}$ vertices at time $t+1, k_{i} \geq 1$, by links which are assigned length $-a$. The $k_{i}$ vertices, $k_{i}>1$, at time-slice $t+1$ will be connected by $k_{i}-1$ consecutive space-like links, thus forming $k_{i}-1$ triangles. Finally the right boundary vertex in the set of $k_{i}$ vertices will be identified with the left boundary vertex of the set of $k_{i+1}$ vertices. In this way we get a total of $\sum_{i=1}^{l}\left(k_{i}-1\right)$ vertices (and also links) at time-slice $t+1$ and the two spatial slices are connected by $\sum_{i=1}^{l} k_{i} \equiv l_{i}+l_{t+1}$ triangles. This is illustrated by Fig. 2 .

The elementary building blocks of a geometry are therefore triangles with one space- and two time-like edges. We define them to be flat in the interior. A consistent way of assigning interior angles to such Minkowskian triangles is described in [7]. The angle between two time-like edges is $\gamma_{t t}=-\arccos \frac{3}{2}$, and between a space- and a time-like edge $\gamma_{s t}=\frac{\pi}{2}+\frac{1}{2} \arccos \frac{3}{2}$, summing up 
to $\gamma_{t}+2 \gamma_{s t}=\pi$. The sum over all angles around a vertex with $j$ incoming and $k$ outgoing timelike edges (by definition $j, k \geq 1$ ) is given by $2 \pi+(4-j-k)$ arccos $\frac{3}{2}$. The regular triangulation of flat Minkowski space corresponds to $j=k=2$ at all vertices. The volume of a single triangle is given by $\frac{\sqrt{3}}{4} a^{2}$.

One may view these geometries as a subclass of all possible triangulations that allow for the introduction of a causal structure. Namely, if we think of all time-like links as being futuredirected, a vertex $v^{\prime}$ lies in the future of a vertex $v$ iff there is an oriented sequence of time-like links leading from $v$ to $v^{\prime}$. Two arbitrary vertices may or may not be causally related in this way.

In Lorentzian quantum gravity we are instructed to sum over all geometries connecting, say, two spatial boundaries of length $L_{1}$ and $L_{2}$, with the weight of each geometry $g$ given by

$$
\mathrm{e}^{i S[g]}, \quad S[g]=\Lambda \int \sqrt{-\operatorname{det} g} \quad(\text { in } 2 \mathrm{~d})
$$

where $\Lambda$ is the cosmological constant. In our discretized model the boundaries will be characterized by integers $l_{1}$ and $l_{2}$, the number of vertices or links at the two boundaries. The path integral amplitude for the propagation from geometry $l_{1}$ to $l_{2}$ will be the sum over all interpolating surfaces of the kind described above, with a weight given by the discretized version of (57). Let us call the corresponding amplitude $G_{\lambda}\left(l_{1}, l_{2}\right)$, which may be represented as the sum

$$
G_{\lambda}\left(l_{1}, l_{2}\right)=\sum_{t=1}^{\infty} G_{\lambda}\left(l_{1}, l_{2} ; t\right)
$$

As already mentioned, $G_{\lambda}\left(l_{1}, l_{2} ; 1\right)$ serves as a transfer matrix for the model. Knowledge of $G_{\lambda}\left(l_{1}, l_{2} ; 1\right)$ allows us to find $G_{\lambda}\left(l_{1}, l_{2} ; t\right)$ by iterating (19) $t$ times. Using standard techniques of generating functions we associate a factor $g=e^{i \lambda}$ with each triangle (not to be confused with the metric $g_{a b}$ ), a factor $x$ with each vertex on the entrance loop and a factor $y$ with each vertex on the exit loop, leading to

$$
G_{\lambda}(x, y ; 1)=\sum_{k=0}^{\infty}\left(g x \sum_{l=0}^{\infty}(g y)^{l}\right)^{k}-\sum_{k=0}^{\infty}(g x)^{k}=\frac{g^{2} x y}{(1-g x)(1-g x-g y)}
$$

Formula (59) is simply a book-keeping device for all possible ways of evolving from an entrance loop of any length in one step to an exit loop of any length. The subtraction of the term $1 /(1-g x)$ has been performed to exclude the degenerate cases where either the entrance or the exit loop is of length zero.

From formula (59) and eqn (20), with $t_{1}=1$, we obtain

$$
G_{\lambda}(x, y ; t)=\frac{g x}{1-g x} G_{\lambda}\left(\frac{g}{1-g x}, y ; t-1\right) .
$$

This equation can be iterated and the solution written as

$$
G_{\lambda}(x, y ; t)=F_{1}^{2}(x) F_{2}^{2}(x) \cdots F_{t-1}^{2}(x) \frac{g^{2} x y}{\left[1-g F_{t-1}(x)\right]\left[1-g F_{t-1}(x)-g y\right]},
$$

where $F_{l}(x)$ is defined iteratively by

$$
F_{t}(x)=\frac{g}{1-g F_{t-1}(x)}, \quad F_{0}(x)=x
$$

Let $F$ denote the fixed point of this iterative equation. By standard techniques one readily obtains

$$
F_{t}(x)=F \frac{1-x F+F^{2 t-1}(x-F)}{1-x F+F^{2 t+1}(x-F)}, \quad F=\frac{1-\sqrt{1-4 g^{2}}}{2 g} .
$$


Inserting (63) in eqn (61), we can write

$$
\begin{aligned}
G_{\lambda}(x, y ; t) & =\frac{F^{2 t}\left(1-F^{2}\right)^{2} x y}{\left(A_{t}-B_{t} x\right)\left(A_{t}-B_{t}(x+y)+C_{t} x y\right)} \\
& =\frac{F^{2 t}\left(1-F^{2}\right)^{2} x y}{\left[(1-x F)-F^{2 t+1}(F-x)\right]\left[(1-x F)(1-y F)-F^{2 t}(F-x)(F-y)\right]},
\end{aligned}
$$

where the time-dependent coefficients are given by

$$
A_{t}=1-F^{2 t+2}, \quad B_{t}=F\left(1-F^{2 t}\right), \quad C_{t}=F^{2}\left(1-F^{2 t-2}\right) .
$$

The combined region of convergence of the expansion in powers $g^{k} x^{l} y^{m}$, valid for all $t$, is

$$
|g|<\frac{1}{2}, \quad|x|<1, \quad|y|<1
$$

We have analyzed the possible continuum limits that may be obtained for expression (64) (see [9] for details). The result is that one only gets an interesting continuum limit if the divergent (unphysical) parts of the bare cosmological constants are taken to be purely imaginary. The Lorentzian form for the continuum propagator is then obtained by an analytic continuation $\Lambda \rightarrow-i \Lambda$ in the renormalized coupling of the resulting Euclidean expressions. At this stage it may seem that we are surreptitiously reverting to a fully Euclidean model. We could of course equivalently have conducted the entire discussion up to this point in the "Euclidean sector", by omitting the factor of $-i$ in the exponential (57) of the action, choosing $\lambda$ positive real and taking all edge lengths equal to 1 . However, from a purely Euclidean point of view there is no reason for restricting the state sum to a subclass of geometries admitting a causal structure. As we will show below, this restriction changes the quantum theory drastically. The associated preferred notion of a discrete "time" allows us to define an "analytic continuation in time". Because of the simple form of the action in two dimensions, the rotation

$$
\int d x d t \sqrt{-\operatorname{det} g_{l o r}} \rightarrow i \int d x d t_{e u} \sqrt{\operatorname{det} g_{e u}}
$$

to Euclidean metrics in our model is equivalent to the analytic continuation of the cosmological constant $\Lambda$.

Rather than discussing in detail how to obtain a continuum limit from (64), let us show how one can derive a differential equation for the continuum $\operatorname{limit} G_{\Lambda}(X, Y ; T)$ of $G_{\lambda}(x, y ; t)$. Inserting (16) in eqn (60) and expanding to first order in the lattice spacing $a$ leads to

$$
a^{\varepsilon-1} \frac{\partial}{\partial T} G_{\Lambda}(X, Y ; T)+\frac{\partial}{\partial X}\left[\left(X^{2}-\Lambda\right) G_{\Lambda}(X, Y ; T)\right]=0
$$

We conclude that an interesting continuum limit exists provided the critical exponent $\varepsilon$ in $T=t a^{\varepsilon}$ is equal to 1 . This value is different from the one obtained for Euclidean gravity where $\varepsilon=1 / 2$. With $\varepsilon=1$, equation (69) becomes a standard first-order partial differential equation which should be solved subject to the boundary condition

$$
G_{\Lambda}(X, Y ; T=0)=\frac{1}{X+Y}
$$

which expresses the condition $G_{\Lambda}\left(L_{1}, L_{2} ; T=0\right)=\delta\left(L_{1}-L_{2}\right)$. The solution is

$$
\begin{aligned}
G_{\Lambda}(X, Y ; T)= & \frac{4 \Lambda \mathrm{e}^{-2 \sqrt{\Lambda} T}}{(\sqrt{\Lambda}+X)+\mathrm{e}^{-2 \sqrt{\Lambda} T}(\sqrt{\Lambda}-X)} \\
& \times \frac{1}{(\sqrt{\Lambda}+X)(\sqrt{\Lambda}+Y)-\mathrm{e}^{-2 \sqrt{\Lambda} T}(\sqrt{\Lambda}-X)(\sqrt{\Lambda}-Y)},
\end{aligned}
$$


or, after an inverse Laplace transformation,

$$
G_{\Lambda}\left(L_{1}, L_{2} ; T\right)=\frac{\mathrm{e}^{-[\operatorname{coth} \sqrt{\Lambda} T] \sqrt{\Lambda}\left(L_{1}+L_{2}\right)}}{\sinh \sqrt{\Lambda} T} \frac{\sqrt{\Lambda L_{1} L_{2}}}{L_{2}} I_{1}\left(\frac{2 \sqrt{\Lambda L_{1} L_{2}}}{\sinh \sqrt{\Lambda} T}\right),
$$

where $I_{1}(x)$ is a modified Bessel function of the first kind. Remarkably, our highly non-trivial expression (72) agrees with the loop propagator obtained from a bona-fide continuum calculation in proper-time gauge of pure $2 \mathrm{~d}$ gravity by Nakayama [11].

This result is clearly different from the one obtained in Euclidean gravity. The "proper time" $T$ has the dimension of $1 / \sqrt{\Lambda}$, while the dimensionality of the analogous quantity in Euclidean gravity, the geodesic distance $R$, is that of $1 / \sqrt[4]{\Lambda}$. This also implies that for large $T$ the average "spatial" volume of a slice at some intermediate time will have an anomalous dimension. In fact, in Euclidean $2 \mathrm{~d}$ quantum gravity we have

$$
G_{\Lambda}^{(e u)}\left(L_{1}, L_{2} ; R\right) \propto \mathrm{e}^{-\sqrt[4]{\Lambda} R} \quad \text { for } \quad R \rightarrow \infty,
$$

from which one can calculate the average two-dimensional volume $V(R)$ in the ensemble of universes with two boundaries separated by a geodesic distance $R$,

$$
\langle V(R)\rangle=-\frac{1}{G_{\Lambda}^{(e u)}\left(L_{1}, L_{2} ; R\right)} \frac{\mathrm{d}}{\mathrm{d} \Lambda} G_{\Lambda}^{(e u)}\left(L_{1}, L_{2} ; R\right) \propto \frac{R}{\Lambda^{3 / 4}} .
$$

For large $R$ we therefore expect the average spatial volume $L_{\text {space }}$ at intermediate $T$ 's to behave like

$$
\left\langle L_{\text {space }}\right\rangle=\frac{\langle V(R)\rangle}{R} \propto \frac{1}{\Lambda^{3 / 4}} .
$$

In the present model, according to (71), the amplitude behaves for large $T$ like

$$
G_{\Lambda}\left(L_{1}, L_{2} ; T\right) \propto \mathrm{e}^{-\sqrt{\Lambda} T},
$$

which implies that the dimension of $T$ in this case is [L]. Instead of (75), we therefore obtain the dependence

$$
\left\langle L_{\text {space }}\right\rangle \propto \frac{1}{\sqrt{\Lambda}} .
$$

This reflects the fact that the quantum space-time of the model does not have an anomalous fractal dimension, and thus differs drastically from the average space-time in the usual theory of two-dimensional Euclidean quantum gravity. Recall that in the classification of the critical behaviour in terms of $(\varepsilon, \eta)$, for purely "kinematical reasons", illustrated in Fig.1, we needed either $(\varepsilon, \eta)=(1 / 2,3 / 2)$ (Euclidean quantum gravity) or $\varepsilon=1, \eta<0$. It can be shown that in the present model $\eta=-1$ (see [9] for details). We do not know if alternative models with different $\eta$ exist.

\subsection{Topology changes of space}

So far in our non-perturbative regularization of Lorentzian $2 \mathrm{~d}$ quantum gravity we have not considered the possibility of spatial topology changes. We will now show that if one includes such topology changes one recovers the usual scaling of Euclidean $2 \mathrm{~d}$ quantum gravity. By a topology change of space in our Lorentzian setting we have in mind the following: a baby universe may branch off at some time $T$ and develop in the future, where it will eventually disappear into the vacuum. However, it is not allowed to rejoin the "parent" universe and thus change the overall 


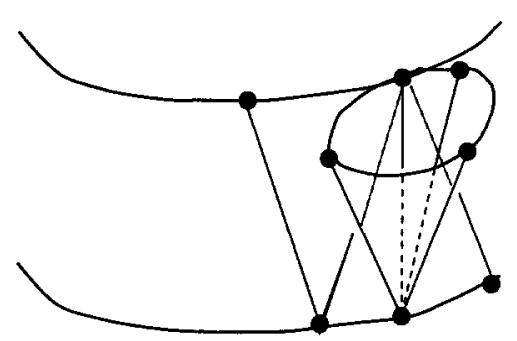

Fig. 3. A "baby universe" branches off locally in one time-step.

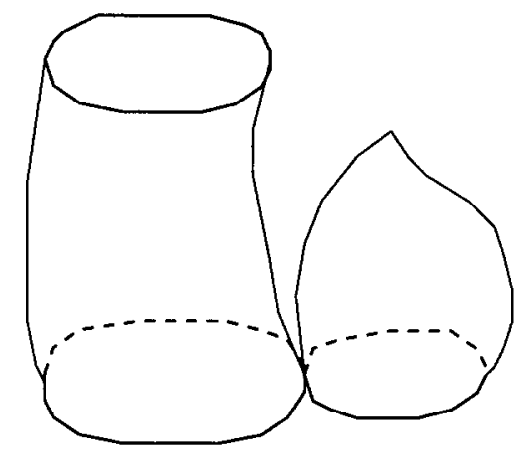

Fig. 4. A "baby universe" is created by a global pinching.

topology of the two-dimensional manifold. This is a restriction we impose to be able to compare with the analogous calculation in usual $2 \mathrm{~d}$ Euclidean quantum gravity.

Our starting point will be the discretized model introduced above. The superscript ${ }^{(b)}$ will indicate the "bare" model without spatial topology changes. There are a number of ways to implement the creation of baby universes, some more natural than others. They all agree in the continuum limit, as will be clear from the general arguments provided below. We mention just two ways of implementing such a change. The first is a simple generalization of the forward step we have used in the original model, where each vertex at time $t$ could connect to $n$ vertices at time $t+1$. We now allow in addition that these sets of $n$ vertices (for $n>2$ ) may form a baby universe with closed spatial topology $S^{1}$, branching off from the rest. The process is illustrated in Fig. 3. An alternative and technically somewhat simpler way to implement the topology change is shown in Fig. 4: stepping forward from $t$ to $t+1$ from a loop of length $l_{1}$ we create a baby universe of length $l<l_{1}$ by pinching it off non-locally from the main branch. We have checked that the continuum limit is the same in both cases. For simplicity we only present the derivation in the latter case.

Accounting for the new possibilities of evolution in each step according to Fig. 4, the new and old transfer matrices are related by

$$
G_{\lambda}\left(l_{1}, l_{2} ; 1\right)=G_{\lambda}^{(b)}\left(l_{1}, l_{2} ; 1\right)+\sum_{l=1}^{l_{1}-1} l_{1} w\left(l_{1}-l, g\right) G_{\lambda}^{(b)}\left(l, l_{2} ; 1\right) .
$$

The factor $l_{1}$ in the sum comes from the fact that the "pinching" shown in Fig. 4 can take place at any of the $l_{1}$ vertices. As before, the new transfer matrix leads to new amplitudes $G_{\lambda}\left(l_{1}, l_{2} ; t\right)$, satisfying

$$
G_{\lambda}\left(l_{1}, l_{2} ; t_{1}+t_{2}\right)=\sum_{l} G_{\lambda}\left(l_{1}, l ; t_{1}\right) G_{\lambda}\left(l, l_{2} ; t_{2}\right)
$$


and in particular

$$
G_{\lambda}\left(l_{1}, l_{2} ; t\right)=\sum_{l} G_{\lambda}\left(l_{1}, l ; 1\right) G_{\lambda}\left(l, l_{2} ; t-1\right)
$$

Performing a (discrete) Laplace transformation of eqn (80) leads to

$$
G(x, y ; g ; t)=\oint \frac{d z}{2 \pi i z}\left[G_{\lambda}^{(b)}\left(x, z^{-1} ; 1\right)+x \frac{\partial}{\partial x}\left(w(x ; g) G_{\lambda}^{(b)}\left(x, z^{-1} ; 1\right)\right)\right] G(z, y ; g ; t-1),
$$

or, using the explicit form of the transfer matrix $G_{\lambda}^{(b)}(x, z ; 1)$, formula (59),

$$
G(x, y ; g ; t)=\left[1+x \frac{\partial w(x, g)}{\partial x}+x w(x, g) \frac{\partial}{\partial x}\right] \frac{g x}{1-g x} G\left(\frac{g}{1-g x}, y ; g ; t-1\right) .
$$

Proceeding as before, we can now derive a differential equation in the continuum limit. We will assume that the disc amplitude scales according to (23), leading to

$$
\begin{aligned}
a^{\varepsilon} \frac{\partial}{\partial T} G_{\Lambda}(X, Y ; T)= & -a \frac{\partial}{\partial X}\left[\left(X^{2}-\Lambda\right) G_{\Lambda}(X, Y ; T)\right] \\
& -a^{\eta-1} \frac{\partial}{\partial X}\left[W_{\Lambda}(X) G_{\Lambda}(X, Y ; T)\right]
\end{aligned}
$$

The first term on the right-hand side of eqn (83) is precisely the one we have already encountered in the model without baby universe creation, while the second term is due to the creation of baby universes. Clearly the case $\eta<0$ (in fact $\eta \leq 1$ ) is inconsistent with the presence of the second term, i.e. the creation of baby universes. However, since $\eta<2$, the last term on the right-hand side of (83) always dominates the first term. Once we allow for the creation of baby universes, this process will completely dominate the continuum limit. In addition we get $\varepsilon=\eta-1$, in agreement with relation (29). Thus $\eta>1$ and we conclude that $\varepsilon=1 / 2, \eta=3 / 2$ are the only possible scaling exponents if we allow for the creation of baby universes. These are precisely the scaling exponents obtained from two-dimensional Euclidean gravity in terms of dynamical triangulations. The topology changes of space have induced an anomalous dimension for $T$. If the second term on the right-hand side of (83) had been absent, this would have led to $\varepsilon=1$, and the time $T$ scaling in the same way as the spatial length $L$. - In summary, eqn (83) leads to the continuum relation

$$
\frac{\partial}{\partial T} G_{\Lambda}(X, Y ; T)=-\frac{\partial}{\partial X}\left[W_{\Lambda}(X) G_{\Lambda}(X, Y ; T)\right],
$$

which coincides with an analogous equation that can be derived directly in $2 \mathrm{~d}$ Euclidean quantum gravity.

\section{DISCUSSION}

In the present article we have demonstrated by explicit calculation that it is possible to formulate a background-independent theory of quantum gravity and still maintain some analogues of concepts from classical gravity, like "rods-and-clocks" measurements of the (quantum) geometry, and propagating test particles to probe the (quantum) geometry. Clearly it is a major limitation that all calculations are performed in two dimensions, and until recently have been limited to the Euclidean sector of quantum gravity. However, any theory of quantum gravity will have to address questions similar the ones we have been dealing with in the context of two-dimensional gravity. 
Our results were two-fold: first it was shown that the generic two-dimensional geometry is fractal, with intrinsic Hausdorff dimension four, and not two. This situation is reminiscent of the random walk representation for the free particle which is of immense importance for our understanding of relativistic quantum field theory. The fact that the fractal dimension of a generic walk is two and not one, as one would naively have expected, underscores the impossibility of associating a classical path with a particle at distances smaller than its inverse mass. However, the concept of intrinsic dimensionality is more complex in two-dimensional quantum gravity, as exemplified by our study of the propagation of test particles. The dimensionality obtained from the return probability of a diffusion process is $t w o$, and not four. Nevertheless, the actual diffusion process is anomalous, in the sense that the average geodesic distance travelled grows only like $\tau^{1 / 4}$, and not $\tau^{1 / 2}$, as a function of the fictitious diffusion time $T$, as would be the case on any non-fractal manifold. However, this anomalous propagation behaviour is counterbalanced by the anomalous volume contained within a radius of $\tau^{1 / 4}$, so that in the end the return probability is the same as on a two-dimensional smooth manifold.

Secondly, we have discussed the relation between Euclidean quantum gravity, defined via a non-perturbative path integral, and a similar construction in the Lorentzian case. Again by explicit calculation, using a simple discretized Lorentzian model where causal structures were included in a natural way, we obtained a continuum limit different from that of Euclidean quantum gravity, but which can again be reproduced by formal continuum calculations. The reason for the difference could be traced to the presence or absence of topology changes of space. In standard classical Lorentzian gravity such topology changes are associated with singular points of the metric structure, but none of the usual principles of quantization tell us whether such configurations should be allowed quantum-mechanically or not. However, we showed that we can only obtain agreement with the theory of Euclidean quantum gravity if we allow for spatial topology changes.

This state of affairs is perfectly acceptable from a physical point of view: unlike in the Lorentzian case, in Euclidean quantum gravity we have no distinction between space- and timelike directions. If we consider the cylinder amplitude, some choices of "time"-slicing may result in baby universe creation as a function of "time", while others may not. The concept of "absence of baby universes" makes only sense in a situation where there is a (class of) preferred time choice(s), as was the case for our discrete Lorentzian geometries. In the Lorentzian case, we had to explicitly allow for the creation of baby universes to obtain a class of geometries matching those summed over in Euclidean quantum gravity. This choice resulted then (up to analytic continuation) in the already known Euclidean quantum theory. Similar intricacies in the relation between Euclidean and Lorentzian quantum gravity are to be expected also in higher dimensions.

Acknowledgement-J. A. acknowledges the support from MaPhySto which is financed by the Danish National Research Foundation.

\section{REFERENCES}

1. F. David, Nucl. Phys. B257 (1985) 45; Nucl. Phys. B257 (1985) 543;

2. J. Ambjørn, B. Durhuus and J. Fröhlich, Nucl. Phys. B257 (1985) 433;

3. V. A. Kazakov, Phys. Lett. B150 (1985) 282.

4. V. A. Kazakov, I. K. Kostov and A. A. Migdal, Phys. Lett. 157B (1985) 295.

5. Y. Watabiki, Prog. Theor. Phys. Suppl. 114 (1993) l.

6. H. Kawai, N. Kawamoto, T. Mogami and Y. Watabiki, Phys. Lett. B306 (1993) 19-26.

7. R. Sorkin, Phys. Rev. D 12 (1975) 385-396; Err. ibid. 23 (1981) 565.

8. T. Jonsson and J. F. Wheater, Nucl.Phys. $B 515$ (1998) 549;

J. D. Correia and J. F. Wheater, Phys. Lett. $\mathbf{B 4 2 2}$ (1998) 76.

9. J. Ambjørn and R. Loll, Non-perturbative Lorentzian Quantum Gravity, Causality and Topology Change, hepth/9805108. 
10. J. Ambjorn, D. Boulatov, J. L. Nielsen, J. Rolf, Y. Watabiki, J. High Energy Phys. 02 (1998) 010.

11. R. Nakayama, Phys. Lett. B325 (1994) 347-353.

12. J. Ambjørn, B. Durhuus J. Fröhlich and P. Orland, Nucl. Phys. B270 (1986) 457;

13. F. David, Mod. Phys. Lett. A5 (1990) 1019.

14. J. Ambjørn, J. Jurkiewicz and Y. M. Makeenko, Phys. Lett. B251 (1990) 517.

15. J. Ambjørn and Y. Watabiki, Nucl. Phys. B445 (1995) 129.

16. J. Ambjørn, J. Jurkiewicz and Y. Watabiki, Nucl. Phys. B454 (1995) 313.

17. S. Havlin and D. Ben-Avraham, Adv. Phys. 36, (1987) 695.

18. J. Ambjørn, B. Durhuus and T. Jonsson, Phys. Lett. B244 (1990) 403.

19. J. Ambjørn, B. Durhuus and T. Jonsson, Quantum Geometry, Cambridge Monographs on Mathematical Physics, Cambridge, 1997.

20. H. Kawai, Nucl. Phys. Proc. Suppl. 26 (1992) 93. 